\title{
Product individual-level harmfulness
}

Addiction Ontology

\section{Source}

Addiction Ontology

Definition: Product harmfulness to an individual.

Comment: This entity relates to the harmfulness for a given individual. It is distinct from population-level harmfulness which refers to harmfulness to a population. Thus a product may have high individual-level harmfulness but low population-level harmfulness if there are very few users in the population. The individual may be a user of the product (e.g. a smoker) or another person (e.g. a family member of a smoker).

This definition was imported from the Addiction Ontology (see https://addictovocab.org/). Comments and suggestions for improvements are welcome using the Qeios review system.

Definitions imported from the Addiction Ontology (AddictO) are what are known as 'ontological definitions'. See this article in Qeios for an explanation https://www.qeios.com/read/YGIF9B.

Ontological definitions can sometimes be hard to read. In those cases we also include an informal definition.

Definitions also often require elaboration to make it clear how they should be used and what they include. In those cases we include a comment.

Also, definitions sometimes require an explanation as to how they came about to help users understand how they relate to alternative definitions. In those cases we include a curator note. 